\title{
And if there was no need of dark energy to explain the acceleration of the expansion of the universe?
}

\author{
Nathalie Olivi-Tran ${ }^{1,2}$ \\ ${ }^{1}$ Laboratoire Charles Coulomb, CNRS, \\ UMR 5221, place Eugene Bataillon, \\ 34095 Montpellier cedex 5, France, \\ ${ }^{2}$ Laboratoire Charles Coulomb, Universite Montpellier 2, \\ place Eugene Bataillon, 34095 Montpellier cedex 5, France \\ email: Nathalie.Olivi-Tran@univ-montp2.fr
}

(Dated: April 18, 2013)

\begin{abstract}
In order to explain the fact that the pressure in the Friedmann equations is negative, only the hypothetical presence of dark energy is used in present theories. But, the dimensions of the pressure $p$ are $f / r^{2}$ and thus $p$ can not account for the acceleration of the expansion of the universe. Indeed, the hypersurface of our universe is threedimensional and curved, so a force has an effect on the universe if it is applied on the universe's boundaries. As these boundaries (hypersurface) correspond to the threedimensional universe itself at time $t$, there must exist a positive force density $f / r^{3}$. The relation between the pressure $p$ (calculated within the Friedmann model) and the force density is a simple derivation with respect to $r$ the space variable. And the derivation of a negative pressure leads to a positive force density.
\end{abstract}

Keywords: existence of dark matter; threedimensional curved universe 


\section{INTRODUCTION}

The question which is arising since the observation of the acceleration of the expansion of the universe is: do we really have to revise the Cosmological Standard Model? Indeed, type Ia Supernovae (SNeIa), anisotropies in the cosmic microwave background radiation (CMBR), and matter power spectrum inferred from large galaxy surveys suggest a revision of this model. Up to now, the most commonly accepted theory implies that cold dark matter (CDM) represents $25.8 \%$ of the universe matter content and dark energy $69.4 \%$ of the energy content (last Planck's results). This theory is explained within the $\Lambda$ CDM model. But, a model would be to analyze the behavior of time during the expansion of the universe. Previous publications suggest that time could be a function of lengths: indeed, by making a simple dimensional analysis of Einstein's field equations, one finds that the dimension of time equals the dimension of a length. A possible explanation of the acceleration of the expansion of the universe would be to consider cosmic time as a function of the radius of curvature of the universe.

\section{THEORY}

Let us make the corresponding calculation within the frame of the Roberston Walker model in the case of the homogeneous vacuum dominated universe. The two equations (Friedmann equations) in this model are:

$$
\left(\frac{\dot{a}}{a}\right)^{2}+\frac{k c^{2}}{a^{2}}-\frac{\Lambda c^{2}}{3}=\frac{8 \pi G}{3} \rho
$$

and

$$
\frac{\ddot{a}}{a}=-\frac{4 \pi G}{3}\left(\rho+\frac{3 p}{c^{2}}\right)+\frac{\Lambda c^{2}}{3}
$$

The cosmological constant term can be omitted if we make the following replacement

$$
\begin{gathered}
\rho \rightarrow \rho+\frac{\Lambda c^{2}}{8 \pi G} \\
p \rightarrow p-\frac{\Lambda c^{4}}{8 \pi G}
\end{gathered}
$$

Therefore the cosmological constant can be interpreted as arising from a form of energy which has negative pressure, equal in magnitude to its (positive) energy density:

$$
p=-\rho c^{2}
$$


Such form of energya generalization of the notion of a cosmological constantis known as dark energy.

In fact, in order to get a term which causes an acceleration of the universe expansion, it is enough to have a scalar field which satisfies

$$
p<-\frac{\rho c^{2}}{3}
$$

Another theory to explain equation (6) is to analyze the pressure $p$.

I make here the hypothesis that our three dimensional universe with physical laws is embedded in a fourdimensional space where no physical law exist. This is consistent with my former publication $[1,2]$.

In the Friedmann model, the pressure $p$ is negative. But the dimensions of $p$ are

$$
f / r^{2}
$$

where $f$ is a constant force (the force due to the energy of vacuum) and $r^{2}$ is a twodimensional surface. If our universe is threedimensional, the pressure would write

$$
f / r^{3}
$$

where $r^{3}$ is the hypersurface of our universe (which corresponds to the universe at time $t$ ). To obtain (7), one has to integrate (8) over $r$, which leads to a negative pressure.

Indeed, the relation between the pressure $p$ and what we call the force density (the force $f$ divided by a threedimensional surface ${ }^{3}$ ) is:

$$
-2 \frac{f}{r^{2}}=\int \frac{f}{r^{3}} d r
$$

So, if the pressure $p=f / r^{2}$ calculated within the Friedmann equations is negative (in the case of a vacuum dominated universe), the corresponding force density $f / r^{3}$ is positive. This seems logical as the energy of vacuum is positive and the force necessary to accelerate the expansion of the universe has to be applied on a threedimensional hypersurface. Indeed, our universe is threedimensional and curved. The hypersurface of the universe corresponds to the universe at a given comoving time $t$. The 'force' of the vacuum can only account for the acceleration of the expansion of the universe if applied to its hypersurface (which is threedimensional).

In conclusion the pressure $p=f / r^{2}$ is negative but the force density $f / r^{3}$ is positive. 


\section{CONCLUSION}

The fact that the pressure $p$ in the second Friedmann equation is negative can be explained by the dimensions of $p$ itself. Indeed, $p$ is a force divided by a twodimensional surface. While only a force divided by a threedimensional surface (a force density) can account for the acceleration of the expansion of the universe. And that because the hypersurface of our universe is threedimensional and curved. The relation between the pressure $p=f / r^{2}$ and the force density $f / r^{3}$ is a simple derivation.

[1] N.Olivi-Tran and P.M.Gauthier, The FLRW cosmological model revisited: Relation on the local time with the local curvature and consequences on the Heisenberg uncertainty principle Adv. Studies Theor. Phys. vol.2 no 6 (2008) 267-270

[2] N.Olivi-Tran What if our three dimensional curved universe was embedded in four dimensional space? Consequences on the EPR paradox Adv. Studies Theor. Phys., Vol. 3, (2009), no. 12, $489-492$

[3] N.Olivi-Tran Dimensional analysis of Einstein's fields equations Adv. Studies Theor. Phys., Vol. 3, (2009), no. 1, 9 - 12 